\title{
REFERENCES
}

1. L. Auslander, Bierberbach's theorem on space groups and discrete uniform subgroups of Lie groups. II, Amer. J. Math. 83 (1961), 276-280.

2. L. Bieberbach, Über die Bewegungsgruppen der Euklidischen Raüme. I, Math. Ann. 70 (1911), 297-336.

3. - Über die Bewegungsgruppen der Euklidischen Raume. II, Math. Ann. 72 (1912), 400-412.

4. S. Helgason, Differential geometry and symmetric spaces, Academic Press, New York, 1962.

Purdue University and

University of California, Berkeley

\section{EXTENSION OF NORMAL FAMILIES OF HOLOMORPHIC FUNCTIONS}

\section{THEODORE J. BARTH ${ }^{1}$}

Let $X$ be a Stein manifold, and let $A$ be an analytic subset of $X$ $\mathrm{A}$ well-known application of Cartan's Theorem $\mathrm{B}$ [2, Théorème 3 p. 52] states that each holomorphic function on $A$ is the restriction of a holomorphic function on $X$. This paper presents a generalization of this application, namely that each normal family of holomorphic functions on $A$ is the restriction of a normal family of holomorphic functions on $X$.

1. Let $X$ be a topological space which is $\sigma$-compact, i.e., the union of a countable family of compact sets. Let $K(X)$ denote the set of all compact subsets of $X$. For $K \in K(X)$ and $f: X \rightarrow C$ define $\|f\|_{K}$ $=\sup \{|f(x)| \mid x \in K\}$. Define

$$
B(X)=\left\{f \mid f: X \rightarrow C,\|f\|_{K}<\infty \text { for all } K \in K(X)\right\} .
$$

Clearly $B(X)$ is a complex vector space, and $\left\{\|\|_{K} \mid K \in K(X)\right\}$ is a family of pseudonorms on $B(X)$ which then becomes a locally convex vector space. Since $X$ is $\sigma$-compact, $B(X)$ is metrizable, and it is readily checked to be a Fréchet space.

Definition. Let $V$ be a vector subspace of $B(X)$. We say that a set $F \subset V$ is normal with respect to $V$ iff every sequence in $F$ has a subsequence which converges in $V$.

Received by the editors October 29, 1964.

1 This research supported in part by the National Science Foundation while the author held a Graduate Fellowship at the University of Notre Dame. 
LEMMA 1. Let $V$ be a vector subspace of $B(X)$. Then $F \subset V$ is normal with respect to $V$ if and only if $\bar{F}$ is a compact subset of $V$.

We say that $F \subset B(X)$ is uniformly bounded on compact sets iff $\sup \left\{\|f\|_{K} \mid f \in F\right\}<\infty$ for all $K \in K(X)$. If $V$ is a vector subspace of $B(X)$ and $F \subset V$ is normal with respect to $V$, then $F$ is uniformly bounded on compact sets.

Definition. Let $V$ be a vector subspace of $B(X)$. We say that the Vitali theorem holds for $V$ iff every set $F \subset V$ which is uniformly bounded on compact sets is normal with respect to $V$.

LEMMa 2. Let $V$ be a vector subspace of $B(X)$. If the Vitali theorem holds for $V$, then $V$ is a closed subset of $B(X)$, i.e., $V$ is a Fréchet space with the induced psudonorms.

Proof. Let $f \in \bar{V}$. Then there exists a sequence $\left\{f_{n}\right\}$ in $V$ such that $f_{n} \rightarrow f$ for $n \rightarrow \infty$. Take $K \in K(X)$. There exists an integer $n_{0}$ such that $\left\|f_{n}-f\right\|_{K}<1$ for $n \geqq n_{0}$. Let $M=\max \left\{\left\|f_{n}\right\|_{K}+1 \mid n=1, \cdots, n_{0}\right\}$. Then $\left\|f_{n}\right\|_{K} \leqq M$ for $n=1,2, \cdots$. Hence $\sup \left\{\left\|f_{n}\right\|_{K} \mid n=1,2, \cdots\right\} \leqq M$ $<\infty$, that is $\left\{f_{n} \mid n=1,2, \cdots\right\}$ is uniformly bounded on compact sets. Since the Vitali theorem holds for $V,\left\{f_{n}\right\}$ has a subsequence which converges in $V$. The limit of this subsequence must be $f$. Hence $f \in V$.

Lemma 3. Let $A$ be a closed subset of the $\sigma$-compact space $X$. Define $R: B(X) \rightarrow B(A)$ by $R(f)=f \mid A$ for $f \in B(X)$. Then $R$ is continuous and linear.

Proof. Clearly $R$ is linear. Each compact subset of $A$ is also compact in $X$. Hence each pseudonorm in $B(A)$ is the restriction of a pseudonorm in $B(X)$. Therefore $R$ is continuous.

Lemma 4. Let $E$ and $F$ be Fréchet spaces, and let $u: E \rightarrow F$ be continuous, linear, and surjective. Let $K$ be a compact subset of $F$. Then there exists a compact subset $K^{\prime}$ of $E$ such that $u\left(K^{\prime}\right)=K$.

Proof. Let $G=u^{-1}(0)$. Then $G$ is a closed linear subspace of $E$, hence $E / G$ is a Fréchet space. Let $r: E \rightarrow E / G$ be the residual map, and define $v: E / G \rightarrow F$ by $v \circ r=u$. Then $v$ is continuous, linear, and bijective. According to $[4, \operatorname{Satz}(1)$, p. 170], $v$ is a topological isomorphism. The lemma then follows from [4, Satz (7), p. 281].

2. If $X$ is a complex space, we define $H(X)$ to be the set of holomorphic functions on $X$. As has been proved by Gunning [3] and Andreotti and Stoll [1, pp. 326-327], the Vitali theorem holds for 
$H(X)$. We say that $F \subset H(X)$ is a normal family of holomorphic functions iff $F$ is normal with respect to $H(X)$.

TheOREM. Let $X$ be a Stein manifold, and let $A$ be an analytic subset of $X$. Let $F=\left\{f_{\lambda} \mid \lambda \in \Lambda\right\}$ be a normal family of holomorphic functions on $A$. Then there exists a normal family $G=\left\{g_{x} \mid \lambda \in \Lambda\right\}$ of holomorphic functions on $X$ such that $g_{\lambda} \mid A=f_{\lambda}$ for $\lambda \in \Lambda$.

Proof. According to the well-known application of Theorem B of Cartan referred to at the beginning of this paper, the restriction map $R: H(X) \rightarrow H(A)$, defined by $R(f)=f \mid A$, is onto. By Lemma $3, R$ is continuous and linear. Since the Vitali theorem holds for $H(X)$ and $H(A)$, they are Fréchet spaces (Lemma 2). Application of Lemma 1 and Lemma 4 completes the proof.

\section{REFERENCES}

1. A. Andreotti and W. Stoll, Extension of holomorphic maps, Ann. of Math. 72 (1960), 312-349.

2. H. Cartan, Varittes analytiques complexes et cohomologie, Colloque sur les fonctions de plusieurs variables, held at Brussels, 1953, pp. 41-55, Thone, Liége, 1953.

3. R. C. Gunning, On Vitali's Theorem for complex spaces with singularities, J. Math. Mech. 8 (1959), 133-141.

4. G. Köthe, Topologische lineare Rüume. I, Springer-Verlag, Berlin, 1960.

UNIVERSITY OF NOTRE DAME 